\title{
Differentiation of time characteristics in subjects with depressive states
}

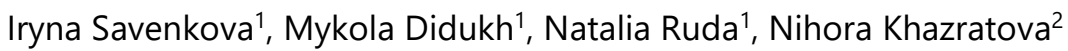

\begin{abstract}
Objective: To distinguish peculiarities of the subjective time perception in subjects with depressive states.

Method: A chronometric sample method using an electronic chronoscope; methods of mathematical statistics: descriptive statistics, methods for determining the mean arithmetic deviation and use, if present, of correlation relations between the value of the individual's subjective time unit and the manifestation level of depressive states; Hamilton scale, Beck questionnaire.

Results: It has been experimentally proved that the forms of depressive states manifestation are consistent with the value of the individual's subjective time unit in the continuous spectrum of $\tau$-types.

Conclusion: A valid difference between quantitative indicators proliferation of various "masks" in certain types of somatic pathology wasn't defined, however, certain trends were observed. Thus, all the main forms of "masks" were present more often in subjects with the cardiovascular system diseases. Algae are approximately equally common in diseases of the gastrointestinal tract, central nervous and cardiovascular systems. "Psychopathological masks" of depression were somewhat more often associated with diseases of the digestive system. Thus, the system of assistance to therapeutic patients with depressive disorders should be based on the principles of a comprehensive approach that integrates various areas of medical and sociopsychological knowledge and practices.
\end{abstract}

Keywords: subjective time unit of the individual, subjective perception of time, depressive states

\section{INTRODUCTION}

Despite the development of knowledge in the field of psychiatry, the problem of diagnosis and treatment of depressive disorders does not lose its relevance. Every year in the world at least 200 million people diagnosed with depression and this figure is constantly increasing (1). Every eight people at least once in a lifetime need special antidepressant therapy. Approximately $2 / 3$ of patients with depression which is diagnosed are subjects of suicidal attempts and $10-15 \%$ commit suicide. $25 \%$ of all patients in the primary health care network suffer from a mental disorder, mainly depressed and anxious. At least $10 \%$ of all users of the primary medical network are undergoing major depression (2).

The help of a family doctor is the primary link in health care. It is at this stage that the primary diagnosis and, if necessary, the further profile diagnostic and medical orientation of patients happens. In $60-70 \%$ of cases in somatic diseases there are violations of the psyche (3). According to many authors, $1 / 3$ of the cases the family doctor is confronted with one or another psychiatric disorder, and only $2-4 \%$ of these patients subsequently turn to a psychiatrist (4-7). This is largely due to the fear of a system of psychiatric care, stigmatization of both mentally ill, and psychiatric services, as well as the historically preconceived prejudice of the population about psychiatric illness. The most difficult in this regard is the problem of psychosomatic and somatopsychic disorders, the psychic radical which the patient is not aware of, as notes I. Savenkova (8). Along with this, mental disorders worsen the course of somatic disease and complicating the clinical picture, forcing doctors to a significant amount of unnecessary research and therapeutic interventions. The most important reason for such situations is the subdepressive and depressive conditions that must be prevented in the early stages of manifestation of psychosomatic disorders. According to I. Savvenkova, $22 \%$ of such

\footnotetext{
1 Sukhomlynskyi Mykolaiv National University, Nikolaev, Ukraine.

2 National University "Lviv Politechnik", Ukraine.
}

Correspondence: Iryna Savenkova

Department of Psychology, Sukhomlynskyi Mykolaiv National University, Nikolaev, Ukraine.

Received: 28 Jan 2019, Accepted: 27 Apr 2019

E-mail: savenkova-1966@ukr.net

(C) 2019 by the authors; licensee Modestum Ltd., UK. This article is an open access article distributed under the terms and conditions of the Creative Commons Attribution License (http://creativecommons.org/licenses/by/4.0/). 
patients are taking up to $50 \%$ of their family doctor's time (9). In view of the huge number of futile research and therapeutic and sometimes surgical interventions, the negative consequences not only concern individual patients, but also the inappropriate economic cost of the health system as a whole (10).

The indicated problems call for the improvement of the system of clinical psychological, diagnostic and therapeutic and prophylactic measures aimed at improving the medical care of people with depressive disorders in the conditions of general-somatic territorial polyclinic and their timely prediction, and accordingly, the prevention. The purpose of the study is to predict the course of depressive states from the view point of the subjective time perception.

In general, the issue of neuropsychic disorders has undergone a well-known transformation from a narrowly clinical to a broad problem of studying the patterns of the formation of normal and abnormal individuals, that is, the problem of studying temperament, character and personality in general. The existing classification of adjacent forms of pathology for today is so fragmented and varied that none of them can be the basis for a single taxonomy of all forms of adjacent states, as R. Brum points out (11).

It should be noted that the time factor of related neuropsychiatric disorders has always been considered approximately. According to I.I. Savvenkova, neurotic disorders have a fairly clear age-frequency (9). Neurosis can not only result in a mental injury. Its formation occurs when the psychological trauma is preceded by the phenomena of over-voltage, fatigue, and the exhaustion of the psyche (12). In recent years, a number of studies have been conducted to prove that the same type of psychological trauma causes different forms of reactive states depending on the individuality of the patient (13). In this case, the new circumstances asthenize the nervous system, and then the slightest cause can lead to neurosis, the content of which manifests a connection with the previously experienced traumatic situation (14). In ones, a psychological trauma never leads to a reactive state, in others - the same injury in similar conditions causes paranoia, or depression, neurasthenia, conversion hysteria, obsessive-compulsive disorder, then, finally, somatoform disfunction of the autonomic nervous system or other variants of somatoform disorders (15).

For the emergence of neurosis, it is necessary that the active psychological trauma is sufficiently strong so to cause a dysfunction of the autonomic nervous system, or that the latter in the given individual was premorbid especially vulnerable (16). O. Kerbikov noted that the mental life of man is formed "... under the influence of the environment, gender, age and temperament" (17). According to the author, the age of the subjects, as a rule, imposes a mark on the degree of neuroticism of patients. The average age at which the pathological development for inhibited individuals has ended is $13.3 \pm 1.4$ years, for hysterical - $14.7 \pm 1.9$ years, for excitations $-22.1 \pm 1.9$ years (18). In this case, the frequency and severity of reactions, as a rule, the more as older the surveyed. Especially pronounced are the phenomena of accent, and, consequently, the risk of occurrence of adjacent disorders that occur after age 21, and even more after 31 years, that is, the factor of time significantly affects the emergence, formation and development of related neuropsychiatric diseases (6). It has long been known that the innate mechanism of the central clock of the individual starts from the moment of birth and continuously measures the course of the time experiencing throughout life. According to the teachings of P. Fress, the life of the individual from birth to death can be broken into a number of periods (19). He highlighted the turning points in life, that is, a certain age in which there are "psychological breaks", and called them nodal points. Many researchers point to the age periodization of human ontogenetic development and isolate the phases of somatic, sexual, neuro-mental, intellectual maturation $(9,20-23)$. These cycles and phases of development are temporary characteristics and can not be independent of the course of the central clocks of the individual.

Thus, from the position of psychology of time it is possible to isolate the zone of the most acute manifestation of adjacent neuropsychic disorders, as well as to calculate the average individual whose chronotype is most vulnerable to the emergence of neurotic, stress-related, and somatoform disorders (24). Using C-frequency, it is possible to accurately calculate the critical period when the nervous breakdown manifests itself in a sharp form with the precision of the individual's subjective clock (7). At the same time it becomes possible to trace the differentiation of their manifestations.

I.I. Savenkova conducted researches that convince that the subjective time unit of the individual and the duration of the individual's biological cycle are the decisive predictive indicators of the psychosomatic disorders manifestation, forms of manifestation of the mental development dysonotogenesis (9).

\section{MATERIALS AND METHODS}

On the basis of the medical center's therapeutic cabinet, 350 patients were examined: 186 (58.1\%) women and 164 (46.9\%) men. The selection of patients was carried out taking into account their diagnosis - the nosological form of psychosomatic illness. The age of the patients ranged from 18 to 67 years, the average age of respondents was $51.6+$ 17.2 years. All patients turned to the clinic for a family doctor on their own initiative. Appeals about the exacerbation of 
chronic diseases were 287 (82.0\%) cases, and with regard to acute conditions - 63 (18.0\%) cases. 189 (54.0\%) patients were at retirement age, $161(46.0 \%)$ were of working age. At that, $133(38.0 \%)$ of the respondents of retirement age continued to work, 56 (16.0\%) of patients who had not reached the retirement age, during the various periods of time did not have work and were on the maintenance of their families. For various reasons, 4 (1.7\%) patients had I, 14 (4.0\%) - II, and 21 (6.0\%) III group of disability. For the duration of the disease, the patients were divided as follows: up to 6 months - 82 (23.4\%), from 7 to 12 months - $6(1.7 \%)$, from 1 to 2 years, 17 (3.8\%), 3 and more years - 245 (70.0\%). All patients who referred to the hospital were examined clinically, using the necessary laboratory and instrumental methods, depending on their underlying disease.

For the verification of somatic pathology, the results of an objective general clinical examination and documented anamnestic information were discussed with doctors of the somatic profile.

For each surveyed, the data list was filled up which included the following:

1) age of the person (indicating the number of years and months at the time of the survey);

2) number, month, year of birth;

3) the type of psychosomatic disorder.

On the basis of the obtained timing results, a table was constructed with the definition of the $\tau$-subjective of the individual's time. For each patient who was examined with the method of chronometric test [12, p.66] " $\tau$-type" was determined, useing the classic way of reproducing intervals to $=2,3,4,5 \mathrm{~s}$, set by the experimenter and reproduced by the electronic chronoscope to the accuracy of $0.001 \mathrm{~s}$. At the same time, each examinee was asked to recreate the duration he experienced limited to two signals - "start" and "finish" in the form of sound that occurs when turning on and stopping the chronoscope. The subjects reproduced the intervals that were set on this chronoscope.

\section{RESULTS}

The individual value of the " $\tau$-type" was calculated according to the formula for each proposed interval:

$$
\tau=\frac{\sum t_{s}}{\sum t_{o}}
$$

where $t_{o}$ is the duration given by the experimenter, and $t_{s}$ - duration of the surveyed person.

The reproduction of each time interval was repeated five times, and then the average value of the subjective unit of time for each subject was counted.

This relation was first proposed by $\mathrm{H}$. Ehrenwald (24) to characterize the individual results of the reproduction method. But if $\mathrm{H}$. Ehrenwald, and then other authors used this relation as an immeasurable coefficient, B.Y. Tsukanov (7) gives it the maintenance of a subjective time unit of the individual. The really given interval $t_{o}$ is the duration experienced in one section of the "arrows of subjective time", and the duration $t_{s}$ is on the second section with respect to $t_{a t}$ which is already in the past. Going out of this, B.Y. Tsukanov (7) comes to the conclusion that the given interval $t_{0}$ in a situation of intense waiting breaks down to a certain number of subjective units of the individual, and the reproduced duration $t_{s}$ consists of that number. This method of reproducing the duration to determine the " $\tau$-type" allows us to solve the chronopsychological prognosing problem of the course of depressive states psychosomatic disorders.

In the process of studying the structure of somatic pathology, respondents received the following data: 5 (1.4\%) dysfunction of the biliary tract, 16 (4.6\%) - cholecystitis, 11 (3.1\%) - chronic blood circulation insufficiency, 40 (11.4\%) ischemic heart disease, 9 (2.6\%) - chronic colitis, 7 (2.0\%) - diabetes mellitus, 32 (9.1\%) - functional heart rhythm disorders with bradycardia, 65 (18.6\%) - myocardial infarction in history, 18 (5.1\%) - arterial essential hypertension, 7 (2.0\%) residual effects after cerebrovascular accident, 73 (20.9\%) - transient ischemic violations of cerebral circulation, $6(1.7 \%)$ - chronic gastritis, 17 (4.9\%) - chronic bronchitis, 11 (3.1\%) - bronchial asthma, 24 (6.9\%) - hypertonic disease, 9 (2.6\%) residual phenomena after ARVI.

Clinical psychiatric examination, use of research criteria $\mathrm{MKH}-10$, Hamilton scale, as well as Beck questionnaire made it possible to detect depressive disorders in 265 (75.7\%) patients. Accordingly, 85 patients (24.3\%) did not find enough evidence necessary for the diagnosis of depressive disorders. These individuals were considered in the study as a control group in relation to respondents with depression.

The statistical processing of the data obtained included the definition of the mean arithmetic deviation and the use, if any, of correlation relations between the value of the subjective unit time of the individual and the level of manifestation of depressive states (Table 1).

The correlation between the value of the subjective time unit of the individual and the level of manifestation of the depressive state (Table 1) shows that the high correlation in both the direct and in reverse order is exactly in the intervals: 


$$
0.94 s<\tau \leq 1.0 s \text { and } 1.0 s<\tau \leq 1.1 s
$$

Table 1: Indicators distribution of the depression level in the continuous spectrum of " $\tau$-types"

\begin{tabular}{|c|c|c|c|c|c|}
\hline \multirow{2}{*}{ « - type» (sec) } & \multirow{2}{*}{ Depression indicator (in points) } & \multicolumn{2}{|c|}{ Quantitative distribution of surveyed (125 = $100 \%)$} & \multirow{2}{*}{ ń $\tau$, where } & \multirow{2}{*}{ ท́ where, $\tau$} \\
\hline & & Surveyed quantity & $\%$ & & \\
\hline $0.7 c \leq \tau<0.8 s$ & 7 & 16 & 4.6 & 0.16 & 0.17 \\
\hline $0.8 c \leq \tau<0.86 s$ & 8 & 6 & 1.7 & 0.17 & 0.19 \\
\hline $0.86 \mathrm{c} \leq \tau \leq 0.94 \mathrm{~s}$ & 12 & 21 & 6 & 0.51 & 0.54 \\
\hline $0.94 \mathrm{c}<\tau \leq 1.0 \mathrm{~s}$ & 28 & 265 & 75.7 & 0.98 & 0.99 \\
\hline $1.0 \mathrm{c}<\tau \leq 1.1 \mathrm{~s}$ & 18 & 42 & 12 & 0.95 & 0.96 \\
\hline
\end{tabular}

NOTE: $\dot{\eta} \tau$, where - the correlation between the value of the unit of time itself and the level of the depressive state;

$\eta$ where, $\tau$ - feedback of the correlation between the level of manifestation of the depressive state and the value of the individual unit time of the individual

\section{DISCUSSION}

Thus, in the course of the study from the perspective of the course of depression chronopsychological prediction, we managed to isolate the zone of least resistance in individuals suffering from depressive disorders. This indicates their belonging to the typological group. Yes, depressed disorders are the place of the least resistance in moderately introverted. So, it has long been known that a moderate introvert is a person with a weak nervous system that has an increased sensitivity, even to weak stimuli. He has expressed introversion and high neuroticism, and inhibition is twice the excitation. Strong stress in a moderately introverted can provoke a depressive disorder.

In analyzing the groups of patients by the forms of somatic pathology, it was found that in patients with depression, hypertonic disease, atherosclerotic encephalopathy, chronic ischemic heart disease with stroke, transient ischemic circulation disorders, functional disorders of heart rhythm with bradycardia $(p<0.05)$ were diagnosed frequently.

The main manifestations, which allowed to refer to the state of depression, were related to mood, intellectual, motor and somatic spheres of patients.

In 20 cases (23.5\% of the number of people with depression), predominantly within the range of mild depression, indistinct symptomatology, polymorphism and extreme variability were observed.

In 23 (27.1\%) patients, there was a paroxysmal feelings of anxiety. It arose suddenly, for no apparent reason, and was often accompanied by suicidal thoughts. 17 afflicted violations were registered. These patients noted a feeling of amount, emptiness, indifference, and a decrease in interests. They felt uncertain, dissatisfied with any of their own actions and deeds.

Patients experienced a gradual increase in non-confidence in themselves, a negative attitude to their own actions, dissatisfaction with their condition. At the same time, on the background of constantly underestimated mood there was an extraordinary emotional vulnerability with the predominant orientation of the module of affective reactions towards the deepening of hypotonia. Patients generally denied having a low mood, however, they often observed affective tension and put forward numerous complaints that essentially responded to a depressed mood. Most patients $(71,86 \%)$ used other (non-depressed) lexical forms.

The following variants of depressive disorders are highlighted.

Hypochondriatic variant. Depressive symptoms were closely related to the experiences of somatic discomfort and were evaluated by the patients as a natural response to a present, although not yet recognized, somatic disease. In marked, persistent cases of viscero-autonomic disorders, which were observed in 17 (20.3\%) cases, patients completely linked their own state with the manifestations of the underlying disease, considering this background of mood the necessary component. In 8 cases, rhythmic deterioration in the somatic area was observed, which was periodically resembled by daily mood fluctuations.

Asthenic variant. Depressive symptoms were presented mainly by adynamic, angry manifestations with complaints of weakness, fatigue, "exhaustion", lack of interest in all aspects of life, besides the disease.

Dysphoric variant. At the forefront was irritability, anger, suspiciousness of patients. In outpatient practice, this option was observed quite often. Such patients can be heard already in the corridor outside the cabinet, since with their appearance there is buzz and shouts. In the office they are also quarrelsome, annoying, angry, during the conversation they behave cautiously, and often aggressively, negatively refer to the doctor's conclusions and to the prescribed treatment. In the course of a long conversation it was possible to "soften" the tension of the patient, but the psychotherapeutic effect in such cases was very unstable.

According to ICD-10 the degree of manifestation of depression in patients was distributed as follows: easy - 60 (71\%) patients; moderate - 19 (22\%) patients; difficult - 6 (7\%) patients. In order to objectify the severity of depressive disorders 
and their quantitative analysis, the Hamilton scale was used to assess depression (NDRS-21). The total score of 7-16 points on the scale was regarded as a small depressive episode, higher than 16 points - as a major depressive episode.

When using this diagnostic tool in order to avoid hyperdiagnosis of depressive disorder or reassess the degree of its severity to somatic points (for example, lack of appetite, fatigue) was given less importance than such items as suicidal thoughts, feelings of guilt, and feeling unlucky. Test results have always been compared with the data obtained during the pathopsychological examination.

The following nosological structure of depression was established: $44.6 \%$ (38) - reactive (mainly nosogenic) depression, 35.3\% (30) - dysthymia, 2.4\% (2) - endogenous, 7.0\% (6) - organic, 7.0\% (6) - somatogenous, 3.5\% (3) iatrogenic depression. The last category included patients with depressive symptoms, which arose in connection with the use of depressant drugs and was leveled after their replacement.

It was found that nosogenic depressions are more common in severe somatic diseases, dysthymia is more often associated with protracted chronic diseases, cyclothymia - with gastroenterological pathology, and vascular - in diseases of the cardiovascular system. Jatrogenic depression occurred at the use of digitalis, corticosteroids, cimetidine.

Depressive states often occurred under the guise of somatic pathology. Among psychopathological masks, anxietyphobic, obsessive-compulsive, hypochondria, neurasthenic disorders were found. Insomnia and hypersomnia were seen as "masks" of biological rhythms violation. Common were somatic "masks" in the form of a vegetative-vascular dystonia syndrome, dizziness, functional disorders of the internal organs, neurodermatitis, skin itching, anorexia, bulimia, impotence, menstrual disorders. Among the "masks" in the form of algea were cardialgia, abdominalia, fibromyalgia, neuralgia, spondyloalgia, pseudo-rheumatic arthralgia. Patho-haematological masks were detected due to disorder of desires, antisocial behavior, hysterical reactions. The presence of "masks" was detected in $69(81.2 \%)$ cases. In 8 (9.4\%) cases, the presence of psychopathological masks was noted, in 3 (3.5\%) cases they were observed in the form of violations of biological rhythm, in 30 (35.3\%) cases - in the form of vegetative-vascular crises, in 22 (25.9\%) - in the form of algea and in $6(7.0 \%)$ cases - in the form of patho-haematological displacements. The indicated "masks" had a different distribution among the selected forms of depressive states: psychopathological masks were more common in cyclothymia, less often - in dysmetic disorders and reactive depression. Violations of biorhythms of sleep with the same frequency were found in patients with reactive, organic and iatrogenic depression. Vegetosomatic masks were predominantly characterized by dysthymia and reactive depression, the same frequency was observed in organic depression and cyclothymia, and only once - with iatrogenesis. Algae "masks" were the priority for reactive states, somewhat less - for dysthymia and with the same frequency were encountered in organic and somatogenic depressions. Patho-haematological masks were prevalent in dysthymia, less frequent in reactive depression and in a single case, with cyclothymia.

There was no significant difference between the quantitative indicators of the distribution of different masks in certain types of somatic pathology, however, certain trends were observed. So, all the main forms of masks were found somewhat more often in diseases of the cardiovascular system organs. Algae are approximately equally common in diseases of the gastrointestinal tract, central nervous and cardiovascular systems. "Psychopathological masks" of depression were somewhat more often associated with diseases of the digestive system.

\section{CONCLUSION}

Thus, the system of assistance to therapeutic patients with depressive disorders should be based on the principles of a comprehensive approach integrating different areas of medical and socio-psychological knowledge and practices.

The results of the study suggest that from the standpoint of chronopsychological prognosing of the depressive states course it becomes possible to isolate the zone of least resistance in individuals suffering from depressive disorders. This indicates their belonging to the typological group. So, depressive disorders are the place of the least resistance in the moderately introverted people with the value of the subjective time unit from $0.94 \mathrm{~s}$ to $1.0 \mathrm{~s}$. Strong stress in a moderately introverted can provoke a depressive disorder.

There is a link between the depression manifestation form and the internal organs psychosomatic diseases. "Depressive masks" are most consistent with diseases of the cardiovascular system, which are the most vulnerable place of manifestation precisely in moderately introverted with the value of the subjective time unit from $0.94 \mathrm{~s}$ to 1 . Algies are second in manifestations of depression and are consistent with diseases of the gastrointestinal tract, central nervous system and cardiovascular system. "Psychopathological masks" occupy the third place in the manifestations of depression and are consistent with the digestive system diseases. 


\section{REFERENCES}

1. Desai G, Chaturvedi SK. Do Diagnostic Criteria for Psychosomatic Research Explain Diagnosis of Medically Unexplained Somatic Symptoms? Psychotherapy and Psychosomatic. 2016;85:121-2. https://doi.org/10.1159/000441063 PMid:26807856

2. Fizeshi O. Historical-System Model of the Development of Primary School in Transcarpathia at the Tum of XIXXX Centuries. Science and education. 2017;12:15-23.

3. Elkelboom EM, Tak LM, Roest AM, Rosmalen JG. A systematic review and meta - analysis of the percentage of revised diagnoses in functional somatic symptoms. Journal of Psychosomatic Research. 2016;88:60-67. https://doi.org/10.1016/j.jpsychores.2016.07.001 PMid:27455914

4. Fava GA, Cosci F, Sonino N. Current Psychosomatic Practice. Psychotherapy and Psychosomatics. 2017;86:13-30. https://doi.org/10.1159/000448856 PMid:27884006

5. Harchenko DM. Personal peculiarities of people with different alexithymic radical. Relevant problems in psychology. 2016;8:127-30.

6. Koffka K. Principles of Gestalt Psychology. Springfield: Merriam-Webster; 2015.

7. Tsukanov BY. Time in human psyche. Odesa: Astroprint; 2000.

8. Savenkova I, Didukh M, Mukhina L, Litvinenko I. Lange biological cycle duration in patients with respiratory organs disorders. Electronic Journal of General Medicine. 2018;15(6):em83. https://doi.org/10.29333/ejgm/99826

9. Savenkova I, Didukh M, Chuhueva I, Litvinenko I. Chronopsychological mental development dysontogenesis prognosing in pre-school children. Electronic Journal of General Medicine. 2019;16(2):em110. https://doi.org/10.29333/ejgm/108595

10. Birren JE. The psychology of ageing. Boston: Little, Brow Company; 2014.

11. Brun R. General treatment of nevrosis. Springfield: Merriam-Webster; 2016.

12. James W. The prinsiples of Psychology. Boston: Little, Brown Company; 2016.

13. Fraisse P. Psychology of Time: conditioning, perception, control, time estimation, concept of time. Basel: E.Reinhard, 2015.

14. Harchenko DM. Psychosomatic disorders in humans with different behavioral activity types. Relevant problems in psychology. 2015;4:284-90.

15. Harchenko DM. Anxiety in people with different alexithymic condition. Relevant problems in psychology. 2015;7:157-9.

16. Henningsen P, Zipfel S, Sattel H, Creed F. Management of Functional Somatic Syndromes and Bodily Distress. Psychotherapy and Psychosomatics. 2018;87(1):12-31. https://doi.org/10.1159/000484413 PMid:29306954

17. Kerbikov AV. About time perception. Moscow: Vysshaya shkola; 2013.

18. Kasimovskaya NA, Fattakhutdinova EV, Khuziakhmetov AN, Kutlin NG, Kostina YA, Kochetkov IG. An attributive component of the condition of people with the diagnosis of "Ischemic heart disease. Effort angina". Electronic Journal of General Medicine. 2018;15(6):em87. https://doi.org/10.29333/ejgm/99830

19. Fress P. Human adaptation to time. Moscow: Progress; 1961.

20. Elkin DG. Experiencing time. Doctors thesis. Odessa: ONU imeni I.I. Mecnikova; 1945.

21. Kuhn TS. The structure of scientific revolutions. Frankfurt: Suhrkamp; 2016.

22. Polunin OV. Psychological research of experiencing the present. Psychology and society. 2013;4:138-43.

23. Shirkavand L, Abbaszadeh A, Borhani F, Momenyan S. Correlation between spiritual well-being with satisfaction with life and anxiety among elderlies suffering from cancer. Electronic Journal of General Medicine. 2018;15(6):em20. https://doi.org/10.29333/ejgm/85501

24. Ehrenwald H. Attempts to time perception of the unconscious ark. New York: Psychologie; 2014.

$\diamond \diamond \diamond \diamond \diamond \diamond \diamond$

http://www.ejgm.co.uk 